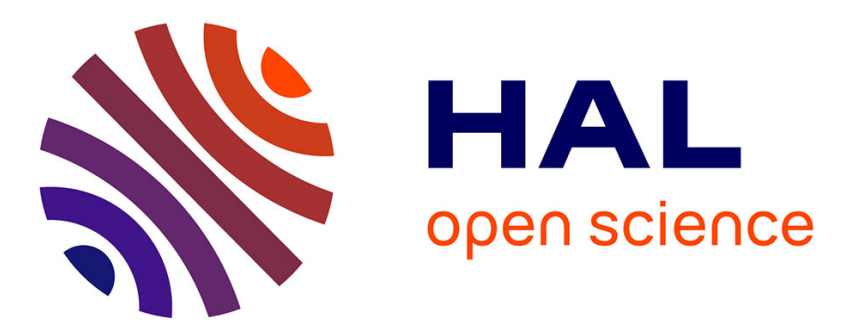

\title{
Optimal location of temporary military zones within civil aviation traffic
}

Ning Wang, Daniel Delahaye, Marcel Mongeau, Alexandre Gondran

\section{To cite this version:}

Ning Wang, Daniel Delahaye, Marcel Mongeau, Alexandre Gondran. Optimal location of temporary military zones within civil aviation traffic. EIWAC 2017, 5th ENRI International workshop on ATM/CNS., Nov 2017, Tokyo, Japan. pp.104-1199, 10.1007/978-981-13-7086-1_7 . hal-01637955

\section{HAL Id: hal-01637955 \\ https://hal-enac.archives-ouvertes.fr/hal-01637955}

Submitted on 18 Nov 2017

HAL is a multi-disciplinary open access archive for the deposit and dissemination of scientific research documents, whether they are published or not. The documents may come from teaching and research institutions in France or abroad, or from public or private research centers.
L'archive ouverte pluridisciplinaire HAL, est destinée au dépôt et à la diffusion de documents scientifiques de niveau recherche, publiés ou non, émanant des établissements d'enseignement et de recherche français ou étrangers, des laboratoires publics ou privés. 


\title{
[EN-A-044] Optimal location of temporary military zones within civil aviation traffic
}

\author{
${ }^{+}$N. Wang, D. Delahaye, M. Mongeau, A. Gondran \\ OPTIM Team / ENAC LAB \\ Université de Toulouse \\ Toulouse, France \\ [ningwang | delahaye | mongeau | gondran]@ recherche.enac.fr
}

\begin{abstract}
In this study, we focus on the problem of locating optimally temporary military zones with the aim of minimizing the number of civil flight trajectories impacted by the military activity, and the distance between the military zone and the military base. We model the military zones by 2D geometry shapes with a vertical extension associated to given flight levels during the temporary zone-activation time window. We propose a mathematical formulation of this problem as a constrained-optimization problem. We then introduce a global optimization methodology based on a simulated annealing algorithm featuring tailored neighborhood-search strategies. This is applied to one day of French traffic involving 8,836 civil flights. The results show that the proposed method is efficient to locate the military zone that is nearest from the military base, while minimizing the impact on civil flight trajectories.
\end{abstract}

Keywords: optimization, military zone, location problem, simulated annealing algorithm

\section{Introduction}

Along with the fast development of aviation technology, more and more aircraft are taking off and landing per hour; air traffic experienced its highest growth over the last five years [1]. The sky becomes more and more congested nowadays. Traditionally, prohibited airspace or no-fly zones are established for security reasons or military activities, where no civil flights are allowed, at all times. However, there are also needs for temporary prohibited zones to perform military training for short periods. The military zone, in this article, is a defined geographical surface above the earth. The location of this kind of military zone can be chosen dynamically according to the existing civil flights, so one minimizes the time spent by the civil aircraft in the military zone. The work in this article takes France as example, and proposes a method to locate a certain shape of military zone during a given period of time in the busy civil aviation traffic.

In France, there are more than 8,000 civil flights in one day; their trajectories cover almost the whole airspace above the French territory, as showed in Fig. 1. An example location of military zone is represented by the green rectangle in this figure, and the position of the military base is the center of the blue circle. Flights passing through this rectangle during the activation time of the military zone are affected and have to be modified. The military zone is to be relocated inside this circle at different time windows so as to minimize the impacts on the current civil flights. Moreover, from the fuel consumption saving point of view, minimizing the distance between the military zone and its military base is also desirable.

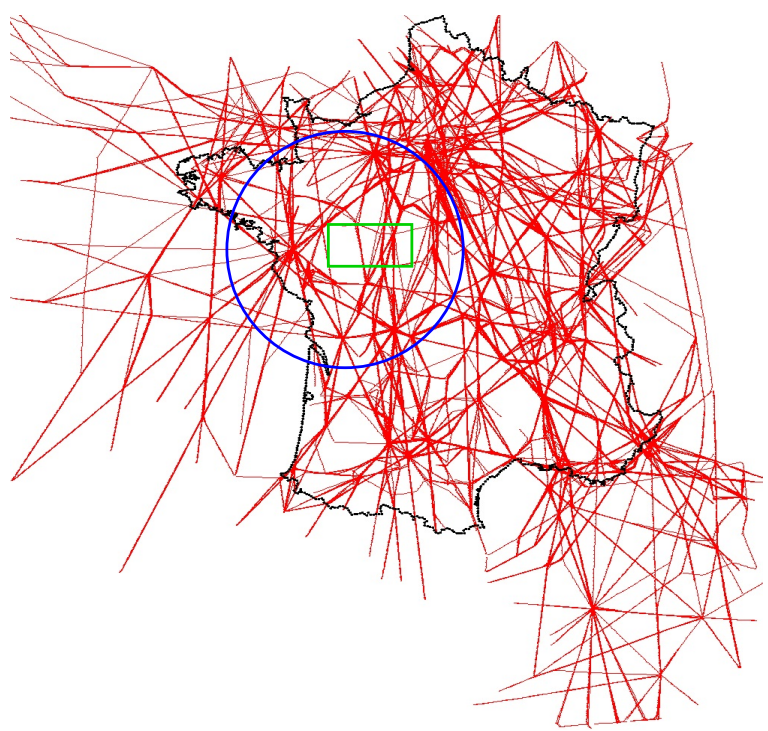

Figure 1: Example of a military zone location (green rectangle) near the military base (center of blue circle) within civil flight trajectories (red lines) over France

To our knowledge, there is no previous published study on the military zone location problem. Nevertheless, one is tempted to consider our problem as a special case of the single facility location problem. 
For this type of problem, "quick and dirty" methods are recommended [2]. For our problem, approximate models are used due to limited input data available for decision makers. Contrary to the classical facility problem, here the facility is considered "toxic", therefore one aims at minimizing the harmful effect (the impact on civil trajectories in our problem) inside the facility (the military zone) coverage range. Another specific feature of our problem is the fact that it deals with continuous candidate locations. The first survey on continous location problems was conducted by $\mathrm{F}$. Plastria; in his work [3], exhaustive topics on continous location theory are discussed. A recent survey on continous location-allocation problems was performed by J. Brimberg [4] in 2008; his work confirms that heuristic methods contributed significantly to improving the methods addressing this type of problem.

The remaining of this article is organized as follows: in Section 2, a mathematical model for the military zone optimal location problem is proposed. Section 3 introduces a simulated annealing algorithm to address this problem with dedicated neighborhood operator. Several test results on the French airspace are presented, and performance of the algorithm is analyzed in Section 4. Finally, conclusions are drawn along with future perspectives for military zone location problems at the end of this article.

\section{Mathematical model}

In this section, we model the military zone by a 2D geometry shape with vertical extension and then formulate our military zone location problem as a constrained optimization problem.

In this study, we aim to locate a military zone for one given military base, the position of this military base is given as input data. To simplify the exposition, the shape of this military zone is defined as a rectangle with a fixed vertical extension, i.e. a cuboid with fixe length $L$, width $W$ and height $H$. It has also a fixed activation time duration $D$. As showed in Fig. 2, it can then be rotated and/or moved around the military base, where $(\rho, \theta)$ are the polar coordinates of this military zone (center of the rectangle), and $\phi$ is the rotation angle of the military zone. We also denote the altitude of the bottom side of this military zone as $z$, considering the military base as the origin, and its starting activation time as $t$. To summarize, the decision variables are $\rho, \theta, \phi, z$ and $t$. In theory, all five variables are continuous variables; In practice, aircraft follow Flight Levels (FL) in order to ensure safe vertical separation. Here we consider discrete elementary altitude shifts $\delta z=5 \mathrm{FL}(1 \mathrm{FL}=100$ feet), and we consider the discrete set $Z:=\left\{z_{\text {min }}+\right.$ $\left.\delta z, z_{\min }+2 \delta z, \ldots, z_{\min }+J \delta z\right\}$ for the possible values of $z$, where $J=\left\lfloor\left(z_{\max }-z_{\min }\right) / \delta z\right\rfloor(\lfloor z\rfloor$ denotes the largest integer greater than or equal to $z), z_{\min }$ and $z_{\max }$ are respectively the lower and upper bounds of altitude constraints for the military zone. To simplify the time allocation, we also consider the discrete time shifts $\delta t=$ 5 minutes, and we define the discrete set $T:=\left\{t_{\text {min }}+\right.$ $\left.\delta t, t_{\text {min }}+2 \delta t, \ldots, z_{\text {min }}+K \delta z\right\}$ for the possible values of $t$, where $K=\left(\left\lfloor t_{\max }-t_{\min }\right) / \delta t\right\rfloor, t_{\min }$ and $t_{\max }$ are respectively the lower and upper bounds of activation time constraints for the military zone. Let us denote $\rho_{\max }$ to be the maximum allowed distance between the center of the military zone and the center of the military base. Let $M Z(x)$ denote the four-dimensional (time +space) military zone defined by an instantiation of our vector of decision variables $x=(\rho, \theta, \phi, z, t)$.

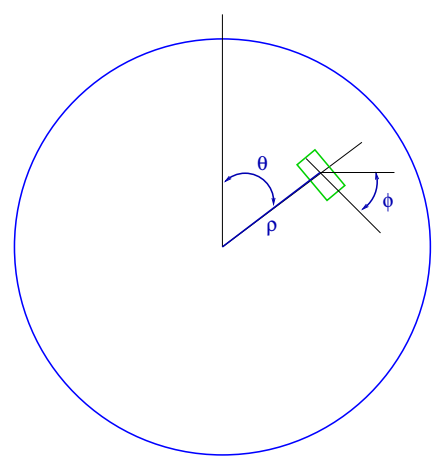

Figure 2: Military zone location model

The given civil flight (4D) trajectories to be avoided are discretized into trajectory sample points $P_{i} \in \mathbb{R}^{4}, i=$ $1,2, \ldots, M$ at intervals of 15 seconds, where $M$ is the total number of sample points for the whole trajectories. In order to minimize the impact of the military zone on the civil traffic, we aim at positioning (in 4D) the military zone so as to minimize the number of trajectory sample points intersecting it, we also wish to minimize the distance between the military zone and the military base $(\rho)$. This is a bi-objective optimization problem that we propose to model as:

$$
\begin{array}{rr}
\underset{x=(\rho, \theta, \phi, z, t)}{\operatorname{minimize}} & f(x)=\sum_{i=1}^{M} 1_{P_{i} \in M Z(x)}+\mu \rho \\
\text { subject to } & \leq \rho \leq \rho_{\max } \\
0 \leq \theta \leq 2 \pi \\
0 \leq \phi \leq 2 \pi \\
z \in Z \\
t \in T
\end{array}
$$

where $1_{P_{i} \in M Z(x)}$ is equal to 1 if $P_{i} \in M Z(x)$ and is equal to zero otherwise. Hence $\sum_{i=1}^{M} 1_{P_{i} \in M Z(x)}$ corresponds to the number of sample trajectory points that lie inside the military zone, and $\mu$ is a weighting parameter whose value is to be set by the decider according to his priority between the two criteria: distance $\rho$ vs. traffic impact. In our tests, we use $\mu=1$ so as to prioritize the impact on civil traffic.

Remark that this model could easily be adapted so as to evaluate the traffic impact by counting rather the 
number of trajectories intersecting the military zone.

When we consider only minimizing traffic impact (case where the user sets $\mu=0$ ), this problem can also be considered as the dual problem of the classical maximum coverage problem involving only one set (one military zone in our case). This one-set situation removes the combinatorial nature of the maximum coverage problem. Nevertheless, instead of the typical discrete candidate sets or a planar (2-dimensional) continuous location problem, we deal here with a 5-dimensional location problem involving 3 continuous location parameters. The difficulty of this continuous optimization problem comes here from the fact that the objective function is non-differentiable and costly to evaluate (a black-box optimization problem), as counting the number of selecting points is time consuming and this computation must be performed at every iteration. This is why we propose here a heuristic resolution method instead of exact approaches.

\section{Simulated annealing algorithm}

Simulated Annealing (SA) is a typical Monte Carlo global optimization algorithm. It is a paradigm that simulates the relationship between the internal energy and the temperature $T$ during the process of metal cooling [5]. Metal is in liquid state at high temperature; its internal energy is at its highest level but this state is highly unstable because of the high disorder of its atomic structure. If a fast cooling procedure is applied, the temper phenomena appears: internal energy quickly drops down but does not converge towards a globally minimal value; the metal has then an amorphous atomic structure with high disorder. However, during a slow cooling process, at each temperature, the internal energy has some probability to reach a locally minimal value. When the temperature reaches room temperature, the internal energy of the metal stabilizes at a minimal value; metal has then a crystal atomic structure.

At the beginning of the cooling process, an initial state $x_{0}$ must be provided. This initial state can be randomly generated or can be particularly chosen to improve the SA performance; for instance, using prior knowledge on the problem, or using any heuristic. The temperature decrease at each iteration $i$ is dealt with some cooling schedule such as: $T_{i}=\alpha^{i} T_{0}$, where $T_{i}$ is the temperature at iteration $i, T_{0}$ is the initial temperature chosen by user and $\alpha \in(0,1)$ is a user-defined parameter. At each iteration/temperature, $N_{t}$ number of transitions (from one solution to another) are performed. At each transition, a neighbor state $x^{\prime}$ of the current state $x$ is generated by some neighborhood operator getNeighbor $(x)$. This new state is then evaluated through the objective function $f$. If the new state is better $\left(f\left(x^{\prime}\right) \leq f(x)\right)$, it is accepted. When the new state is worse than the current state, it is accepted with some probability $\operatorname{proba}_{i}\left(x, x^{\prime}\right)$ related to the current temperature and the objective-function degradation. The most-used acceptance probability for a minimization problem is:

$$
\left.\operatorname{proba}_{(} x, x^{\prime}\right)=\exp \frac{\left(f\left(x^{\prime}\right)-f(x)\right)}{T}
$$

The idea is that a bad transition is more likely to be accepted at high temperatures.

After acceptance or rejection of the new state, the algorithm proceeds to the next transition. The algorithm stops when $T_{i}$ goes below some pre-defined final temperature $T_{f}$ (for instance, we use $T_{f}=0.0001 T_{0}$ in our tests), or some pre-defined target objective-function value is reached, or after performing $N$ iterations, where $N$ is the maximum number of iterations decided by user. $\mathrm{SA}$ is given in Algorithm 1:

Algorithm 1: Simulated annealing algorithm for a minimization problem

$$
\begin{aligned}
& T \leftarrow T_{0}, x \leftarrow x_{0} \\
& \text { while } T>T_{f} \text { do } \\
& \text { for } i=1, \ldots, N_{t} \text { transitions do } \\
& x^{\prime} \leftarrow \operatorname{getNeighbor}(x) \\
& \text { if } f\left(x^{\prime}\right) \leq f(x) \text { then } x \leftarrow x^{\prime} \text {; } \\
& \text { else } x \leftarrow x^{\prime} \text { with probability } \\
& \exp \frac{f\left(x^{\prime}\right)-f(x)}{T_{i}} \\
& \text { end } \\
& T \leftarrow \alpha T \text {; } \\
& \text { end } \\
& \text { return } X \text {; }
\end{aligned}
$$

The neighborhood operator getNeighbor $(x)$ for our problem returns a neighbor state $x^{\prime}\left(\rho^{\prime}, \theta^{\prime}, \phi^{\prime}, z^{\prime}, t^{\prime}\right)$ of current state $x(\rho, \theta, \phi, z, t)$ through the following five functions, chosen randomly with, in our tests, an identical probability, 1/5:

- Translate $(x)$ : return $x^{\prime}$, where $\rho^{\prime}$ is chosen randomly in $\left[0, \rho_{\max }\right]$, and $\theta^{\prime}=\theta, \phi^{\prime}=\phi, z^{\prime}=z$, $t^{\prime}=t$.

- $\operatorname{Rot}_{\theta}(x)$ : Rotate the military zone around the military base, return $x^{\prime}$ where $\theta^{\prime}$ is chosen randomly in $[0,2 \pi]$, and $\rho^{\prime}=\rho, \phi^{\prime}=\phi, z^{\prime}=z$, $t^{\prime}=t$.

- $\operatorname{Rot}_{\phi}(x):$ Rotate the military zone around its center, return $x^{\prime}$ where $\phi^{\prime}$ is chosen randomly in $[0,2 \pi]$, and $\rho^{\prime}=\rho, \theta^{\prime}=\theta, z^{\prime}=z, t^{\prime}=t$.

- ShiftAltitude $(x)$ : return $x^{\prime}$, where $z^{\prime}$ is chosen randomly in $Z$, and $\rho^{\prime}=\rho, \theta^{\prime}=\theta, \phi^{\prime}=\phi, t^{\prime}=t$.

- ShiftActivationTime $(x)$ : return $x^{\prime}$, where $t^{\prime}$ is chosen randomly in $T$, and $\rho^{\prime}=\rho, \theta^{\prime}=\theta, \phi^{\prime}=\phi$, $z^{\prime}=z$.

\section{Simulation results in the French airspace}

In this section, we apply the simulated annealing algorithm on one day of French traffic involving 
8,836 civil flight trajectories, discretized into $1,851,029$ trajectory sample points at intervals of 15 seconds. We first present the input military zone parameters used in the numerical tests and the values we choose for the user-defined parameters involved in the SA algorithms. Then, numerical results obtained are presented and analyzed.

The input military zone parameters are listed in Table 1:

Table 1: Input military zone parameters

\begin{tabular}{|c|c|c|}
\hline parameter & value & unit \\
\hline length $(L)$ & 40 & $\mathrm{NM}$ \\
width $(W)$ & 20 & $\mathrm{NM}$ \\
height $(H)$ & 5,000 & feet \\
$z_{\min }$ & 10,000 & feet \\
$z_{\max }$ & 30,000 & feet \\
$\delta z$ & 1,000 & feet \\
$J$ & 20 & - \\
$D$ & 120 & minutes \\
$t_{\min }$ & $10 \mathrm{AM}$ & - \\
$t_{\max }$ & $12 \mathrm{AM}$ & - \\
$\delta t$ & 5 & minutes \\
$K$ & 24 & - \\
\hline
\end{tabular}

The SA algorithm parameters are chosen as follows:

- Temperature decreasing parameter: $\alpha=0.95$

- Number of transitions for each temperature: $N_{t}=$ 200

- Initial temperature: $T_{0}$ calculated empirically at $80 \%$ acceptance rate

- Final temperature: $T_{f}=0.0001 T_{0}$

Table 2: Simulation results with length $L=40 \mathrm{NM}$ with different values of $\rho_{\max }$

\begin{tabular}{|c|c|c|c|}
\hline$\rho_{\max }(\mathrm{NM})$ & 100 & 200 & 400 \\
\hline nbTrajs & 1339 & 1778 & 2113 \\
nbPts & 100,741 & 171,331 & 226,316 \\
\hline nbPts* & 0 & 0 & 0 \\
$\Delta$ nbPt & 0 & 0 & 0 \\
$\rho^{*}(\mathrm{NM})$ & 53.2 & 51.1 & 50.8 \\
$\Delta \rho^{*}(\mathrm{NM})$ & 2.3 & 3.8 & 1.4 \\
$C P U(\mathrm{~s})$ & 114 & 299 & 285 \\
\hline
\end{tabular}

We first apply the algorithm for three different values of the range $\rho_{\max }: 100 \mathrm{NM}, 200 \mathrm{NM}$, and $400 \mathrm{NM}$. We run the SA algorithms 10 times with the same parameter setting, average results will be computed over these 10 runs. In each case, the algorithm finds a military base with possible minimal impact on the civil flights: zero impact on the civil flights. The results obtained are presented in Table 2, where nbTrajs and $n b P t s$ represent respectively the number of trajectories and sample points in the base range that can potentially be impacted, $\overline{n b P t s^{*}}$ is the average minimal number of impacted trajectory sample points, $\overline{\rho^{*}}$ is the average minimal distance of the military zone found by the SA algorithm, $\triangle n b P t^{*}$ is the average deviation of $n b P t s^{*}$ for 10 runs, $\Delta \rho^{*}$ is the average deviation of $\rho^{*}, C P U$ represents the average $\mathrm{SA}$ algorithm run time in seconds.

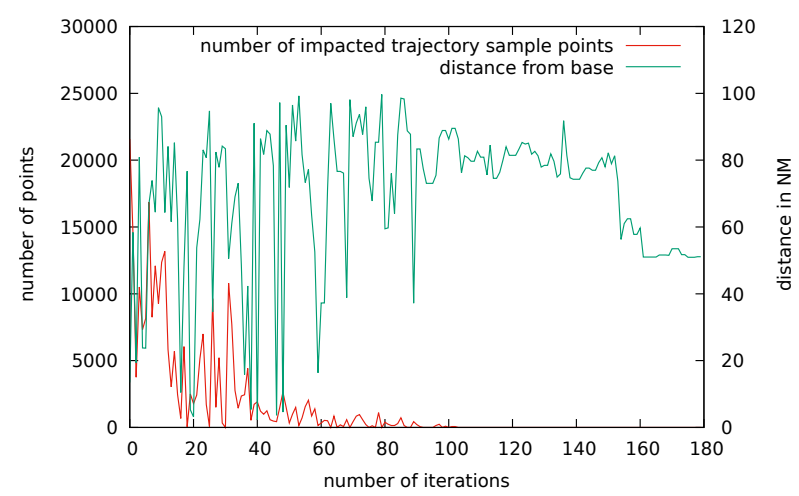

Figure 3: Evolution of the two criteria for $\rho_{\max }=100$ NM

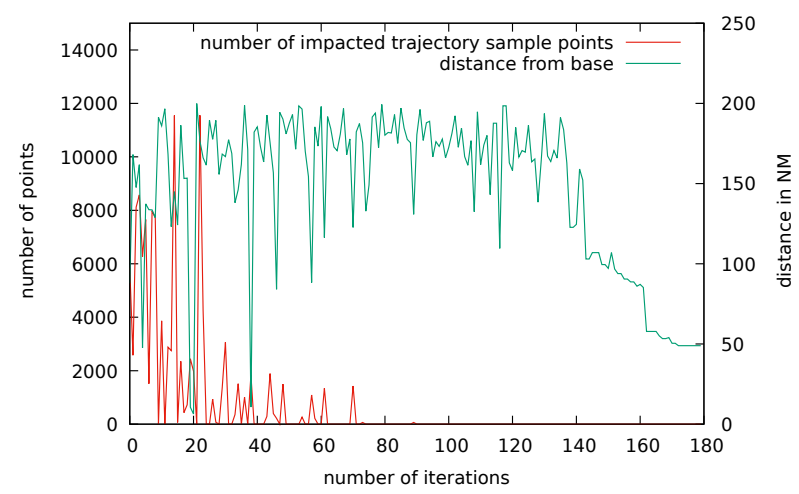

Figure 4: Evolution of the two criteria for $\rho_{\max }=200$ NM

Fig. 3, Fig. 4 and Fig. 5 display the number of impacted trajectory sample points and the distance between the military zone and its base at each iteration of the SA algorithm for three different ranges $\left(\rho_{\max }\right)$. In the objective function, the second criteria (distance from the military base is expressed in NM) is a small value in comparison with the first criterion (number of impacted points), since we chose to set the weighting parameter to $\mu=1$. As a consequence, we observe that the SA algorithm concentrates first on minimizing the first criteria (impact), and then pursues with minimizing the distance from the base while keeping the first criterion at its minimal value. The nearest military zone found is $51 \mathrm{NM}$ away from the base for the range $100 \mathrm{NM}$. The objective function value converges faster with the increasing range limits, and the nearest military zone found is situated at $49 \mathrm{NM}$ from the base for ranges 200 NM and 400 NM. The SA algorithm finds better locations 
when the range gets larger, because the probability of finding a location with zero impact on the civil flights increases with the range.

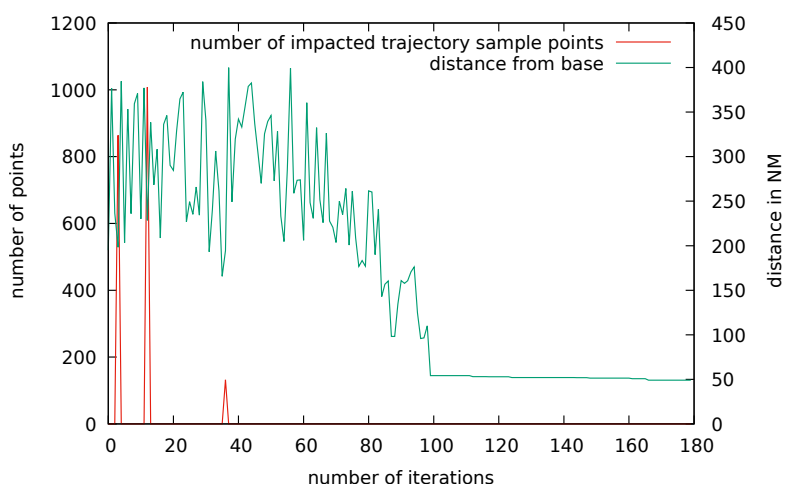

Figure 5: Evolution of the two criteria for $\rho_{\max }=400$ NM

To analyze whether the algorithm is sensitive to the shape and size of the military zone, we test different shapes and sizes of the military zone by modifying the input parameters: length, width, height and activation duration. The results obtained by SA for shape change and size reduction of the military zone are similar to those obtained above. However, if we increase the size of the military zone, SA may fail to find a military zone with zero impact on civil trajectories under small range limitations. Table 3 displays the results obtained for example when doubling the length of the military zone: $\Delta n b P t^{*}$ and $\Delta \rho^{*}$ are relatively large for the range of $100 \mathrm{NM}$ since for 3 runs out of 10 , the algorithm finds a military location near from the base but impacting hundreds of trajectory sample points.

Table 3: Simulation results with length $L=40 \mathrm{NM}$ with different values of $\rho_{\max }$

\begin{tabular}{|c|c|c|c|}
\hline$\rho_{\max }(\mathrm{NM})$ & 100 & 200 & 400 \\
\hline$n b T r a j$ & 1,561 & 1,932 & 2,117 \\
$n b P t$ & 131,272 & 188,288 & 229,178 \\
\hline$n b P t^{*}$ & 62.4 & 0 & 0 \\
$\Delta n b P t^{*}$ & 88 & 0 & 0 \\
$\rho^{*}(\mathrm{NM})$ & 83.8 & 104 & 93.1 \\
$\Delta \rho^{*}(\mathrm{NM})$ & 18.8 & 2.4 & 0.18 \\
$C P U(\mathrm{~s})$ & 281 & 843 & 596 \\
\hline
\end{tabular}

\section{Conclusion and perspectives}

As the air traffic becomes more and more crowded, it becomes more difficult to perform military activities in the air without impacting civil flights. In this article, we studied the location problem of a temporary military zone within civil aviation traffic dedicated for short-time military activities. The military zone was modeled by a 2D geometry shape, with vertical extension associated to given specified flight levels, that has the flexibility to move in space and time. A constrained-optimization formulation was proposed, it aims at minimizing both the impact of military activities on civil flights, and the distance between the military zone and the military base. A simulated annealing algorithm was designed to address this black-box optimization problem. Numerical experiments were conducted on instances involving one day of French traffic. The results indicates that the proposed methodology is a viable decision-aid tool to search for the nearest military zone having no impact on the traffic, as in our tests none of the existing 8,836 civil flights were impacted under different range limitations. Various tests on different input parameters show that the SA algorithm is robust and not sensitive to the shape and size changes of the military zone.

In future work, one may address the dynamic version of this location problem, in which the position of the center of the military zone may change with time during the given activation time window. This is relevant for instance when one deals with the military zone encompassing an in-flight supply plane. Another promising track of research could envisage using a deterministic black-box optimization methods such as those proposed in [6, 7]. Finally, the military location problem may be combined with a real-time flight-trajectory conflict prediction and resolution problem. In this case, conflict resolution approaches will be applied on the trajectories impacted by the chosen military zone.

\section{Acknowledgments}

This work has been supported by Civil Aviation University of China and the program of China Scholarships Council.

\section{References}

[1] Leahy, J., "Airbus global market forecast for 2016-2035”, Airbus Group, 2015. URL: http://www.aircraft.airbus.com/market/global-marketforecast-2016-2036.

[2] Moradi, E. and Bidkhori, M.,'Single facility location problem", in Facility Location: Concepts, Models, Algorithms and Case Studies, Zanjirani, F. R., and Hekmatfar, M. (editors), Physica-Verlag HD, Heidelberg, 2009. p.37-68.

[3] Plastria, F., "Continuous location problems: Research, results and questions." In Facility location: A survey of applications and methods, Z. Drezner (editor), Springer, 1995. p.85-127.

[4] Brimberg, J., Hansen, P., Mladenovic, N., and Salhi, S., "A survey of solution methods for the continuous location allocation problem." International Journal of Operations Research (2008): 1-12 
[5] "Simulated Annealing: Theory and Applications", Laarhoven, P. J. M. and Aarts, E. H. L. (editors), Kluwer Academic Publishers, Norwell, MA, USA, 1987.

[6] Conn, Andrew R., Scheinberg, Katya, and Vicente, Luis N., "Introduction to Derivative-Free Optimization", Society for Industrial and Applied Mathematics, Philadelphia, PA, USA, 2009

[7] Audet, C., and Hare, W., "Derivative-Free and Blackbox Optimization", Springer Series in Operations Research and Financial Engineering, Springer International Publishing, 2018, in press.

\section{Copyright}

The authors of this article confirm that they and their institution, hold copyright of all original material included in their paper. They also confirm they have obtained permission, from the copyright holder of any third party material included in their paper, to publish it as part of their paper. The authors grant full permission for the publication and distribution of their paper as part of the EIWAC2017 proceedings or as individual off-prints from the proceedings. 